\title{
Chaotic scattering in a molecular system
}

\author{
Alex M. Barr, Kyungsun Na, and L. E. Reichl \\ Center for Complex Quantum Systems and Department of Physics, The University of Texas at Austin, Austin, Texas 78712 \\ Christof Jung \\ Instituto de Ciencias Fisicas, Universidad Nacional Autonoma de Mexico, Av. Universidad 1001, 62251 Cuernavaca, Mexico
}

(Received 7 October 2008; published 19 February 2009)

\begin{abstract}
We study the classical dynamics of bound state and scattering trajectories of the chlorine atom interacting with the HO molecule using a two-dimensional model in which the HO bond length is held fixed. The bound state system forms the $\mathrm{HOCl}$ molecule and at low energies is predominantly integrable. Below dissociation a number of bifurcations are observed, most notably a series of saddle-center bifurcations related to a 2:1 and at higher energies 3:1 resonance between bend and stretch motions. At energies above dissociation the classical phase space becomes dominated by a homoclinic tangle which induces a fractal distribution of singularities in all scattering functions. The structure of the homoclinic tangle is examined directly using Poincare surfaces of section as well as indirectly through its influence on the time delay of the scattered chlorine atom and the angular momentum of the scattered $\mathrm{HO}$ molecule.
\end{abstract}

DOI: 10.1103/PhysRevE.79.026215

PACS number(s): 05.45.Ac, 34.35.+a

\section{INTRODUCTION}

When an atomic or molecular system has a classical counterpart, the nonlinear dynamics of the classical system can serve as a template for understanding the quantum dynamics of the atomic or molecular system [1,2]. The presence of chaos in the classical phase space can cause a range of energy eigenstates of the quantum system to spread throughout the chaotic region of the classical phase space. If bifurcations, leading to the formation of new periodic orbits, occur in the classical system, new classes of energy eigenstates can form in the quantum system [3-5]. Analogous effects can occur in open systems such as intense laser-driven atomic or molecular systems. For example, the interaction of the extra electron in the chlorine ion $\mathrm{Cl}^{-}$, with neutral $\mathrm{Cl}$, can be modeled in terms of an inverted Gaussian potential with a single bound state. When an intense laser field is applied to the quantum system, the bound state becomes destabilized and forms a quasibound state (a long-lived but unstable quantum state). As the laser intensity increases, a second quasibound state is formed which helps to stabilize the electron in the presence of the intense laser field [6]. The formation of the second quasibound state has been linked directly to a bifurcation and the formation of new periodic orbits in the underlying classical phase space at energies well above the dissociation energy of the electron [7]. It is interesting that, if the same electron interacts with a Morse potential with a single bound state, no analogous bifurcation occurs in the classical phase space and no second quasibound state forms in the quantum system with increasing laser intensity [7-9].

Another effect of intense laser radiation acting on an atomic system is the formation of chaotic invariant sets (homoclinic or heteroclinic tangles) that extend far into the asymptotic region of the classical phase space and induce a fractal set of singularities into all dynamical quantities related to the scattering process [10-14]. Complicated bifurcations and the resulting new periodic orbits are often embedded in the chaotic sea that is associated with the heteroclinic tangles and, potentially, can induce the formation of addi- tional quasibound states in the quantum system $[7,15]$.

In this paper, we consider the classical scattering behavior in a molecular system, where the internal degrees of freedom of the molecule drive each other in a manner analogous to the effect of a laser driving the electron described above. We will focus on the scattering properties of the $\mathrm{HOCl}$ molecule. Joyeux et al. [3] have shown that $\mathrm{HOCl}$ vibrational dynamics, for energies at or below the $\mathrm{HO}+\mathrm{Cl}$ dissociation energy, can be well described by a model in which the HO bond is held fixed. Indeed, the HO bond only begins to play an important role in $\mathrm{HOCl}$ dynamics at energies well above the $\mathrm{HO}+\mathrm{Cl}$ dissociation energy where the $\mathrm{HClO}$ isomer can be formed. The work of Joyeux et al. focused on the bound state energy regime below dissociation. The focus of this paper is the $\mathrm{HO}+\mathrm{Cl}$ classical scattering dynamics above dissociation, although we also discuss some aspects of the bound state regime.

Below dissociation $\mathrm{HOCl}$ dynamics is largely integrable. It is only slightly below and above dissociation that large scale chaos becomes mixed with regions of regular dynamics in the classical phase space. Quantum mechanically, the $\mathrm{HO}+\mathrm{Cl}$ dissociation process involves decay rates with values that range over several orders of magnitude $[4,16]$, indicating that there likely is a complex collection of quasibound states that determine the dissociation of the molecule. It has been shown in $[7,17]$ that quasibound states can find support on both periodic orbits above dissociation and on heteroclinic or homoclinic tangles in the chaotic region of the open system. For this reason, the purpose of this paper is to provide a classical analysis of $\mathrm{HO}+\mathrm{Cl}$ scattering dynamics.

In Sec. II we review the derivation of the kinetic energy for $\mathrm{HOCl}$ in the case of zero total angular momentum and introduce the two-dimensional potential energy surface (PES) used in this study. The principle periodic orbits as well as periodic orbits born out of subsequent bifurcations are discussed in Sec. III. The evolution of these periodic orbits is followed from low energy all the way to dissociation or until the orbit becomes engulfed in chaos. Section IV examines scattering dynamics. The homoclinic tangle which dominates 


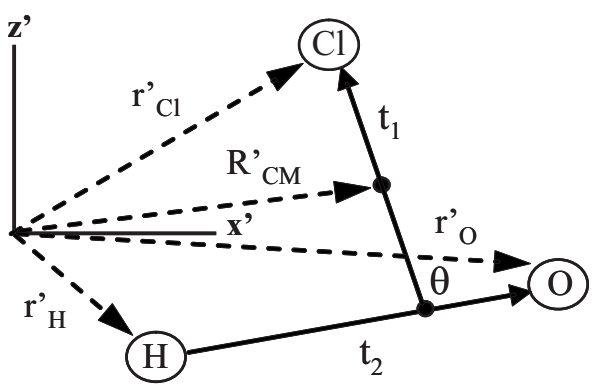

(a)

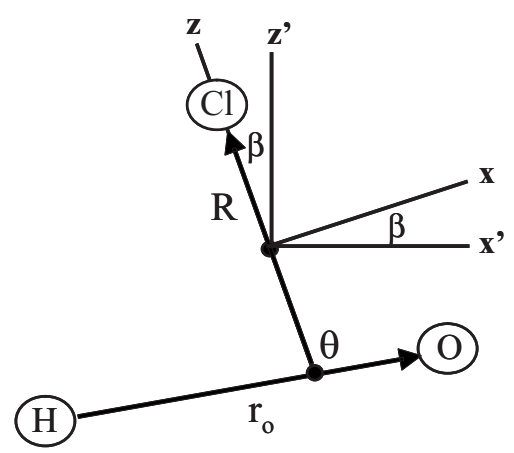

(b)

FIG. 1. (a) Relationship between laboratory frame coordinates and Jacobi vectors $\mathbf{t}_{1}, \mathbf{t}_{2}$, and $\theta$. (b) Body-fixed and laboratory axes differ by an angle $\beta$ so that $\mathbf{t}_{1}$ always lies along the body $z$ axis.

phase space above dissociation is shown and its influence on various scattering functions is examined. In Sec. V we summarize our results and make some concluding remarks.

\section{TWO-DIMENSIONAL MODEL OF HOCL}

$\mathrm{HOCl}$ consists of $\mathrm{H}, \mathrm{O}$, and $\mathrm{Cl}$ atoms with masses $m_{\mathrm{H}}, m_{\mathrm{O}}$ and $m_{\mathrm{Cl}}$. In the subsequent analysis, we shall assume that the total angular momentum of the molecule, $\mathbf{L}_{\text {tot }}$, is zero so all motion occurs in the plane of the molecule. We introduce laboratory coordinates $\left(x^{\prime}, y^{\prime}, z^{\prime}\right)$ and body-fixed coordinates $(x, y, z)$ whose origin is the center of mass of the molecule. Because $\mathbf{L}_{\text {tot }} \equiv 0$, we can assume that all of the dynamics occurs in the $\left(x^{\prime}, z^{\prime}\right)$ and $(x, z)$ planes and that any angular momentum vectors generated by internal rotations of the molecule (such vectors must add to zero) lie along the $y^{\prime}$ and $y$ axes in the laboratory and body-fixed frames, respectively. A sketch of $\mathrm{HOCl}$ relative to the laboratory and body frames is shown in Fig. 1.

Let $\mathbf{t}_{1}$ be a vector of length $R$ that connects the center of mass of $\mathrm{HO}$ to $\mathrm{Cl}$ and $\mathbf{t}_{2}$ a vector of length $r_{o}$ that connects $\mathrm{H}$ to $\mathrm{O}$. The angle between $\mathbf{t}_{1}$ and $\mathbf{t}_{2}$ is $\theta$ where $\theta=0$ for the linear configuration $\mathrm{H}-\mathrm{O}-\mathrm{Cl}$. The center of mass of the molecule lies along $\mathbf{t}_{1}$ a distance $m_{d} R / M$ from the $\mathrm{Cl}$ atom, where $m_{d}=m_{\mathrm{O}}+m_{\mathrm{H}}$ and $M=m_{\mathrm{Cl}}+m_{\mathrm{O}}+m_{\mathrm{H}}$. Let $\mathbf{r}_{\mathrm{Cl}}^{\prime}$, $\mathbf{r}_{\mathrm{H}}^{\prime}$, and $\mathbf{r}_{\mathrm{O}}^{\prime}$ denote the displacement of the $\mathrm{Cl}, \mathrm{H}$, and $\mathrm{O}$ atoms from the laboratory frame origin. Let $\mathbf{R}_{\mathrm{c} . \mathrm{m} .}=\left(m_{\mathrm{Cl}} \mathbf{r}_{\mathrm{Cl}}^{\prime}+m_{\mathrm{O}} \mathbf{r}_{\mathrm{O}}^{\prime}\right.$ $\left.+m_{\mathrm{H}} \mathbf{r}_{\mathrm{H}}^{\prime}\right) / M$ denote the displacement of the center of mass of $\mathrm{HOCl}$ from the laboratory frame origin. Then,

$$
\begin{gathered}
\mathbf{r}_{\mathrm{Cl}}^{\prime}=\mathbf{R}_{\mathrm{c} . \mathrm{m} .}+\frac{m_{d}}{M} \mathbf{t}_{1}, \quad \mathbf{r}_{\mathrm{H}}^{\prime}=\mathbf{R}_{\mathrm{c} . \mathrm{m} .}-\frac{m_{\mathrm{Cl}}}{M} \mathbf{t}_{1}-\frac{m_{\mathrm{O}}}{m_{d}} \mathbf{t}_{2}, \\
\mathbf{r}_{\mathrm{O}}^{\prime}=\mathbf{R}_{\text {c.m. } .}-\frac{m_{\mathrm{Cl}}}{M} \mathbf{t}_{1}+\frac{m_{\mathrm{H}}}{m_{d}} \mathbf{t}_{2} .
\end{gathered}
$$

It is now straightforward to show that the kinetic energy is given by

$$
\begin{aligned}
T & =\frac{m_{\mathrm{Cl}}}{2} \dot{\mathbf{r}}_{\mathrm{Cl}}^{\prime 2}+\frac{m_{\mathrm{O}}}{2} \dot{\mathbf{r}}_{\mathrm{O}}^{\prime 2}+\frac{m_{\mathrm{H}}}{2} \dot{\mathbf{r}}_{\mathrm{H}}^{\prime 2} \\
& =\frac{M}{2} \dot{\mathbf{R}}_{\mathrm{c} . \mathrm{m} .}^{2}+\frac{m_{\mathrm{Cl}} m_{d}}{2 M} \dot{\mathbf{t}}_{1}^{2}+\frac{m_{\mathrm{H}} m_{\mathrm{O}}}{2 m_{d}} \dot{\mathbf{t}}_{2}^{2} .
\end{aligned}
$$

We assume that $\mathbf{t}_{1}$ lies along the body $z$ axis and that the body $y$ axis is directed perpendicular to the plane of the molecule. We can then write $\mathbf{t}_{1}=R \hat{\mathbf{z}}$ and $\mathbf{t}_{2}=r_{o} \sin (\theta) \hat{\mathbf{x}}$ $+r_{o} \cos (\theta) \hat{\mathbf{z}}$. We further assume that the body frame $(x, z)$ axes make an angle $\beta$ with respect to the laboratory frame $\left(x^{\prime}, z^{\prime}\right)$ axes so that if the two frames rotate relative to one another, the angular velocity of rotation is $\dot{\beta} \hat{\mathbf{y}}$. Taking the HO bond length to be fixed at $r_{o}$ so that $\dot{r}_{o}=0$, the time derivatives of vectors $\mathbf{t}_{1}$ and $\mathbf{t}_{2}$ become [18]

$$
\begin{gathered}
\dot{\mathbf{t}}_{1}=\dot{R} \hat{\mathbf{z}}+\dot{\beta} \hat{\mathbf{y}} \times \mathbf{t}_{1}, \\
\dot{\mathbf{t}}_{2}=r_{o} \dot{\theta} \cos (\theta) \hat{\mathbf{x}}-r_{o} \dot{\theta} \sin (\theta) \hat{\mathbf{z}}+\dot{\beta} \hat{\mathbf{y}} \times \mathbf{t}_{2} .
\end{gathered}
$$

Substituting Eqs. (3) into Eq. (2) and dropping the center-ofmass motion we obtain

$$
T=\frac{\mu_{1}}{2}\left(\dot{R}^{2}+R^{2} \dot{\beta}^{2}\right)+\frac{\mu_{2}}{2}\left(r_{o}^{2} \dot{\theta}^{2}+2 r_{o}^{2} \dot{\theta} \dot{\beta}+r_{o}^{2} \dot{\beta}^{2}\right),
$$

where $\mu_{1}=m_{\mathrm{Cl}} m_{d} / M=\frac{595}{52} u$ and $\mu_{2}=m_{\mathrm{O}} m_{\mathrm{H}} / m_{d}=\frac{16}{17} u$ with $u$ the atomic mass unit.

In terms of the canonical momenta $p_{R}=\frac{\partial T}{\partial \dot{R}}=\mu_{1} \dot{R}, p_{\theta}=\frac{\partial T}{\partial \dot{\theta}}$ $=\mu_{2} r_{o}^{2}(\dot{\theta}+\dot{\beta})$, and $p_{\beta}=\frac{\partial T}{\partial \dot{\beta}}=\mu_{2} r_{o}^{2}(\dot{\theta}+\dot{\beta})+\mu_{1} R^{2} \dot{\beta}$, the kinetic energy takes the form

$$
T=\frac{p_{R}^{2}}{2 \mu_{1}}+\frac{p_{\theta}^{2}}{2 \mu_{2} r_{o}^{2}}+\frac{p_{\theta}^{2}}{2 \mu_{1} R^{2}}-\frac{p_{\theta} p_{\beta}}{\mu_{1} R^{2}}+\frac{p_{\beta}^{2}}{2 \mu_{1} R^{2}} .
$$

The total angular momentum of the molecule is given by

$$
\mathbf{L}_{\text {tot }}=\mu_{1} \mathbf{t}_{1} \times \dot{\mathbf{t}}_{1}+\mu_{2} \mathbf{t}_{2} \times \dot{\mathbf{t}}_{2}=p_{\beta} \hat{\mathbf{y}} .
$$

The condition $\mathbf{L}_{\mathrm{tot}}=0$ requires that $p_{\beta}=0$. Note, however, that $\mathbf{L}_{1}=\mu_{1} \mathbf{t}_{1} \times \dot{\mathbf{t}}_{1}=\mu_{1} R^{2} \dot{\beta} \hat{\mathbf{y}}$ and $\mathbf{L}_{2}=\mu_{2} \mathbf{t}_{2} \times \dot{\mathbf{t}}_{2}=\mu_{2} r_{o}^{2}(\dot{\theta}+\dot{\beta}) \hat{\mathbf{y}}$ need not be zero so that $\mathrm{Cl}$ and $\mathrm{HO}$ can rotate relative to one another.

With $p_{\beta}=0$ the Hamiltonian for $\mathrm{HOCl}$ can be written

$$
H=\frac{p_{R}^{2}}{2 \mu_{1}}+\frac{p_{\theta}^{2}}{2 \mu_{2} r_{o}^{2}}+\frac{p_{\theta}^{2}}{2 \mu_{1} R^{2}}+D_{e} V(R, \theta)=E,
$$

where $V(R, \theta)$ is the potential energy of interaction between the atoms in the $\mathrm{HOCl}$ molecule, and $E$ is the total energy of the system. The quantity $r_{o}=1.85 a_{0}$, where $a_{0}=0.52917$ $\times 10^{-10} \mathrm{~m}$ is the Bohr radius. The quantity $D_{e}$ 
TABLE I. Coefficients for $\mathrm{HOCl}$ potential.

\begin{tabular}{lcccc}
\hline \hline$j$ & $b_{j, 0}$ & $b_{j, 1}$ & $b_{j, 2}$ & $b_{j, 3}$ \\
\hline 0 & $-1.08247 \times 10^{-1}$ & $-1.45971 \times 10^{1}$ & $-1.54498 \times 10^{2}$ & $-6.85639 \times 10^{2}$ \\
1 & $5.29617 \times 10^{0}$ & $-9.05238 \times 10^{-1}$ & $1.60231 \times 10^{2}$ & $2.87197 \times 10^{3}$ \\
2 & $-7.97769 \times 10^{-1}$ & $3.54588 \times 10^{0}$ & $-2.02327 \times 10^{2}$ & $2.59771 \times 10^{2}$ \\
3 & $6.77553 \times 10^{-1}$ & $3.65210 \times 10^{1}$ & $-7.71768 \times 10^{2}$ & $-4.69590 \times 10^{3}$ \\
4 & $-1.94808 \times 10^{0}$ & $5.52491 \times 10^{-1}$ & $9.98501 \times 10^{2}$ & $-9.97391 \times 10^{3}$ \\
5 & $-8.26970 \times 10^{-1}$ & $-5.96464 \times 10^{1}$ & $1.18547 \times 10^{3}$ & $1.15942 \times 10^{4}$ \\
6 & $1.72804 \times 10^{0}$ & $-5.68401 \times 10^{0}$ & $-1.50514 \times 10^{3}$ & $8.43773 \times 10^{3}$ \\
7 & $-1.49334 \times 10^{0}$ & $4.00405 \times 10^{1}$ & $1.66305 \times 10^{2}$ & $-7.87303 \times 10^{3}$ \\
$j$ & $b_{j, 4}$ & $b_{j, 5}$ & & $b_{j, 7}$ \\
\hline 0 & $-1.27119 \times 10^{4}$ & $-1.32484 \times 10^{5}$ & $-5.33837 \times 10^{5}$ & $-7.76511 \times 10^{5}$ \\
1 & $1.01590 \times 10^{4}$ & $-1.41755 \times 10^{4}$ & $3.34596 \times 10^{4}$ & $5.09881 \times 10^{5}$ \\
2 & $4.47516 \times 10^{3}$ & $-9.60566 \times 10^{4}$ & $-5.47422 \times 10^{5}$ & $-5.91095 \times 10^{5}$ \\
3 & $1.03525 \times 10^{5}$ & $7.47435 \times 10^{5}$ & $1.41575 \times 10^{6}$ & $1.16233 \times 10^{6}$ \\
4 & $-1.23790 \times 10^{5}$ & $-3.71698 \times 10^{5}$ & $-6.89122 \times 10^{5}$ & $-3.46593 \times 10^{5}$ \\
5 & $-1.02553 \times 10^{4}$ & $-5.72320 \times 10^{5}$ & $-5.10810 \times 10^{5}$ & $-1.31129 \times 10^{6}$ \\
6 & $1.40564 \times 10^{5}$ & $5.48984 \times 10^{5}$ & $1.07966 \times 10^{6}$ & $6.00759 \times 10^{5}$ \\
7 & $-2.10729 \times 10^{4}$ & $-1.54580 \times 10^{5}$ & $-5.36741 \times 10^{5}$ & $2.66176 \times 10^{5}$ \\
\hline \hline
\end{tabular}

$=20312.3 \mathrm{~cm}^{-1}=2.518 \mathrm{eV}=4.035 \times 10^{-19} \mathrm{~J}$ is the dissociation energy of $\mathrm{Cl}$ from the $\mathrm{HO}$ complex. The potential energy is given by

$$
\begin{aligned}
V(R, \theta)= & \left(\frac{1}{2.51841}\right)\left(\frac{1}{2}\right)\left[1+\tanh \left(6-R_{\mathrm{ClO}}\right)\right] \\
& \times \sum_{i=0}^{7} \sum_{j=0}^{7} b_{i, j}\left(\left\{1-\exp \left[-0.8\left(R_{\mathrm{ClO}}-3.2\right)\right]\right\}^{i+1}-1\right) \\
& \times\left\{1-\exp \left[-0.1\left(R_{\mathrm{ClH}}-4.0\right)\right]\right\}^{j}
\end{aligned}
$$

where $R_{\mathrm{ClO}}$ and $R_{\mathrm{ClH}}$ are the distances between $\mathrm{Cl}$ and $\mathrm{O}$ and $\mathrm{Cl}$ and $\mathrm{H}$, respectively. These distances are measured in units of Bohr radii and are related to $R$ and $\theta$ by the triangle equations

$$
R_{\mathrm{ClO}}=\sqrt{\left(\frac{m_{\mathrm{H}}}{m_{d}}\right)^{2} r_{o}^{2}+R^{2}-2\left(\frac{m_{\mathrm{H}}}{m_{d}}\right) r_{o} R \cos (\theta)}
$$

and

$$
R_{\mathrm{ClH}}=\sqrt{\left(\frac{m_{\mathrm{O}}}{m_{d}}\right)^{2} r_{o}^{2}+R^{2}+2\left(\frac{m_{\mathrm{O}}}{m_{d}}\right) r_{o} R \cos (\theta)} .
$$

The coefficients $b_{i, j}$ are given in Table I. The potential energy minimum, $V=-1$, occurs at $\left(\bar{R}_{\mathrm{ClO}}=3.20957, \bar{R}_{\mathrm{ClH}}=3.99748\right)$. From Eqs. (9) and (10) this corresponds to $\left(R_{m}=3.23201\right.$, $\left.\theta_{m}=1.34660\right)$. A contour plot of the potential energy is shown in Fig. 2.

The two-dimensional (2D) potential energy in Eq. (8) is derived from the three-dimensional (3D) potential energy studied extensively in [4] by setting $R_{\mathrm{HO}}=r_{o}=1.85 a_{0}$. Joyeux et al. have studied $\mathrm{HOCl}$ using a similarly derived 2D potential energy starting with the 3D potential energy in [19].
Comparing the 2D PESs, Fig. 2 and Fig. 1 in [3], both surfaces are symmetric about $\theta=0$, have a high wall at $\theta=\pi$ and a saddle point and potential hill along $\theta=0$. These potential barriers at $\theta=\pi$ and $\theta=0$ rise high above the $\mathrm{HO}+\mathrm{Cl}$ dissociation energy and play a significant role in the scattering dynamics of Sec. IV. The PES saddle point in [3] occurs approximately $1000 \mathrm{~cm}^{-1}$ lower in energy than the saddle point in our surface. Small quantitative differences between the surfaces such as this lead to some differences in the evolution of bound periodic orbits. These differences will be discussed in Sec. III as they arise. $\mathrm{HOCl}$ also has an isomer, $\mathrm{HClO}$, which is not included in either 2D PES. The isomerization barrier lies high above the $\mathrm{HO}+\mathrm{Cl}$ dissociation en-

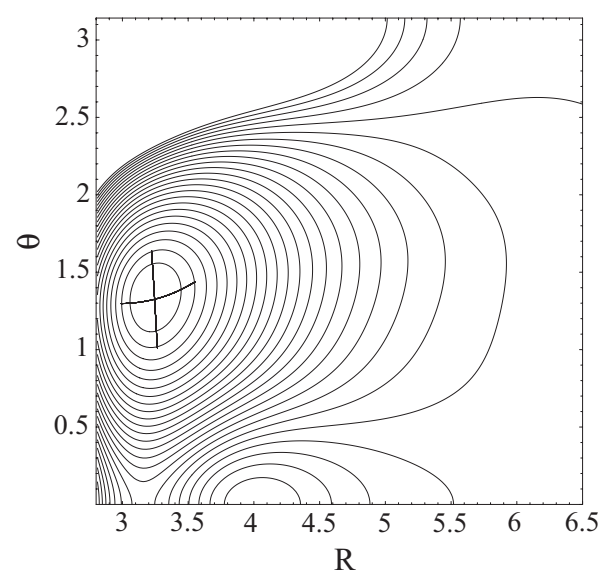

FIG. 2. Contour plot of the 2D PES. Contour spacing is $1000 \mathrm{~cm}^{-1}$ with the highest contour at $25000 \mathrm{~cm}^{-1}$. HOCl equilibrium is $R_{m}=3.23201, \theta_{m}=1.34660$, and $E=0$. Also shown are periodic orbits [R] and [B] at $E=2000 \mathrm{~cm}^{-1}$. 
ergy [20] and the isomer does not play any role in the bound or scattering dynamics considered here.

We can write the Hamiltonian in dimensionless form if we write energies in terms of $D_{e}$ and lengths in terms of $a_{0}$. Then $H=D_{e} H^{\prime}, \quad r_{o}=r_{o}^{\prime} a_{0}, R=R^{\prime} a_{0}, p_{R}=\hbar p_{R}^{\prime} / a_{0}, p_{\theta}=\hbar p_{\theta}^{\prime}$, and $t$ $=\hbar t^{\prime} / D_{e}$ where $\hbar=1.05457 \times 10^{-34} \mathrm{~J} \mathrm{~s}$ is Planck's constant divided by $2 \pi$. If we now drop the primes on the dimensionless quantities we obtain

$$
H=\frac{1}{2}\left(\frac{p_{R}^{2}}{\Delta_{R}^{2}}+\frac{p_{\theta}^{2}}{\Delta_{\theta}^{2}}+\frac{p_{\theta}^{2}}{\Delta_{R}^{2} R^{2}}\right)+V(R, \theta),
$$

where $\Delta_{R}^{2}=\frac{\mu_{1} a_{0}^{2} D_{e}}{\hbar^{2}}=1930.43$ and $\Delta_{\theta}^{2}=\frac{m_{d}}{\mu_{1}} r_{o}^{2} \Delta_{R}^{2}=543.44$.

\section{BOUND STATE PERIODIC ORBITS}

The bound state motion of the $\mathrm{HO}-\mathrm{Cl}$ system can be divided into two energy regimes: Energies below the saddle point energy, $0 \leqslant E \leqslant 17010 \mathrm{~cm}^{-1}$ and energies between the saddle point energy and the dissociation energy, $17010 \mathrm{~cm}^{-1} \leqslant E \leqslant 20312 \mathrm{~cm}^{-1}$. We will discuss these two regimes separately.

\section{A. Below the saddle point}

The dynamics of the $\mathrm{HO}-\mathrm{Cl}$ system, below the saddle point, is largely integrable in character. The angular motion of $\mathrm{Cl}$ about $\mathrm{HO}$, which predominantly involves the coordinate $\theta$, we call "bend" motion, and is close to harmonic for the entire range of energies from the potential minimum to the saddle point. The radial motion of $\mathrm{Cl}$ relative to $\mathrm{HO}$, which predominantly involves the coordinate $R$, we call "stretch" motion. This motion is anharmonic with a frequency that decreases with energy for energies from $E=0$ up to about $E=12000 \mathrm{~cm}^{-1}$ (all energies are given relative to the PES minimum).

Periodic orbits (POs) are located using a shooting method. Starting at a point in phase space, $\mathbf{x}_{0}$ $=\left\{R(0), \theta(0), p_{R}(0), p_{\theta}(0)\right\}$, this point is integrated forward in time through a period $T$ to find $\mathbf{x}(T)$. If $\mathbf{x}(T)-\mathbf{x}_{0}=0$ then $\mathbf{x}_{0}$ is a point on a PO. If $\mathbf{x}(T)-\mathbf{x}_{0} \neq 0$, a Newton-Raphson root finding algorithm is used to find a new $\mathbf{x}_{0}^{\prime}$ to bring $\mathbf{x}(T)^{\prime}$ $-\mathbf{x}_{0}^{\prime}$ closer to zero [21]. If the initial $\mathbf{x}_{0}$ lies within a stable island surrounding a PO this algorithm generally converges on the PO within a few iterations. In addition, this method automatically constructs the Monodromy matrix, $\partial \mathbf{x}(T) / \partial \mathbf{x}_{0}$, whose trace and eigenvalues give information about the stability and bifurcations of the PO [21-23].

At very low energies, near the potential minimum, there exist two principle periodic orbits corresponding to normal mode motion. These principle POs are shown in Fig. 2 at energy $E=2000 \mathrm{~cm}^{-1}$. The PO [R], extends approximately parallel to the body $z$ axis and consists primarily of changes in $R$, the distance of the $\mathrm{Cl}$ atom from the $\mathrm{HO}$ center of mass. The PO [B], consists primarily of rotation of $\mathrm{Cl}$ about the HO complex. At low energies bend and stretch motions are only weakly coupled. As energy is increased [R] and [B] initially extend further along their original (normal mode) directions. Because the potential is anharmonic in $R$ the fre-

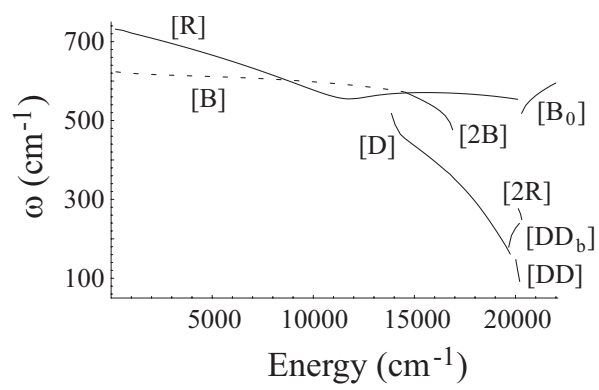

FIG. 3. Bifurcation diagram showing frequency versus energy for principle POs as well as stable POs born at bifurcations. The dotted line for the $[\mathrm{B}]$ periodic orbit represents $\frac{1}{2} \omega_{[\mathrm{B}]}$. This is done to more clearly illustrate the $2: 1$ Fermi resonance between $\omega_{[\mathrm{B}]}$ and $\omega_{[\mathrm{R}]}$.

quency of $[\mathrm{R}]$ decreases approximately linearly with increasing energy as shown in Fig. 3. Near $E=7500 \mathrm{~cm}^{-1}$ the frequency of $[\mathrm{R}]$ becomes equal to one-half the frequency of [B]. This results in a 2:1 Fermi resonance between bend and stretch motions. Once [R] tunes into resonance, bend and stretch motions become strongly coupled for this PO causing [R] to abandon its original direction and take on progressively more of a horseshoe shape as energy is added to this PO. This behavior can be clearly seen in Figs. 4(a) and 4(b). $\mathrm{PO}[\mathrm{B}]$ is unaffected by the Fermi resonance and continues to extend along its original direction as its energy is increased.

In Figs 5(a) and 5(b), we show Poincaré surfaces of section (SOS) of the stretch and bend motions for energy $E$ $=14000 \mathrm{~cm}^{-1}$. The stretch SOS is generated by plotting
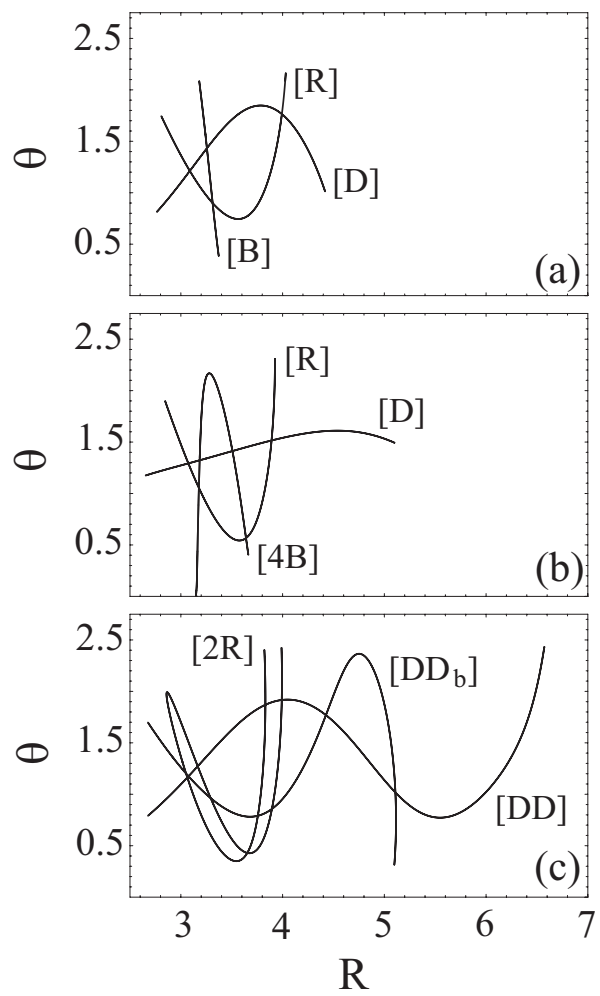

FIG. 4. Stable POs at (a) $E=14000 \mathrm{~cm}^{-1}$, (b) $E=17020 \mathrm{~cm}^{-1}$, and (c) $E=20150 \mathrm{~cm}^{-1}$. 

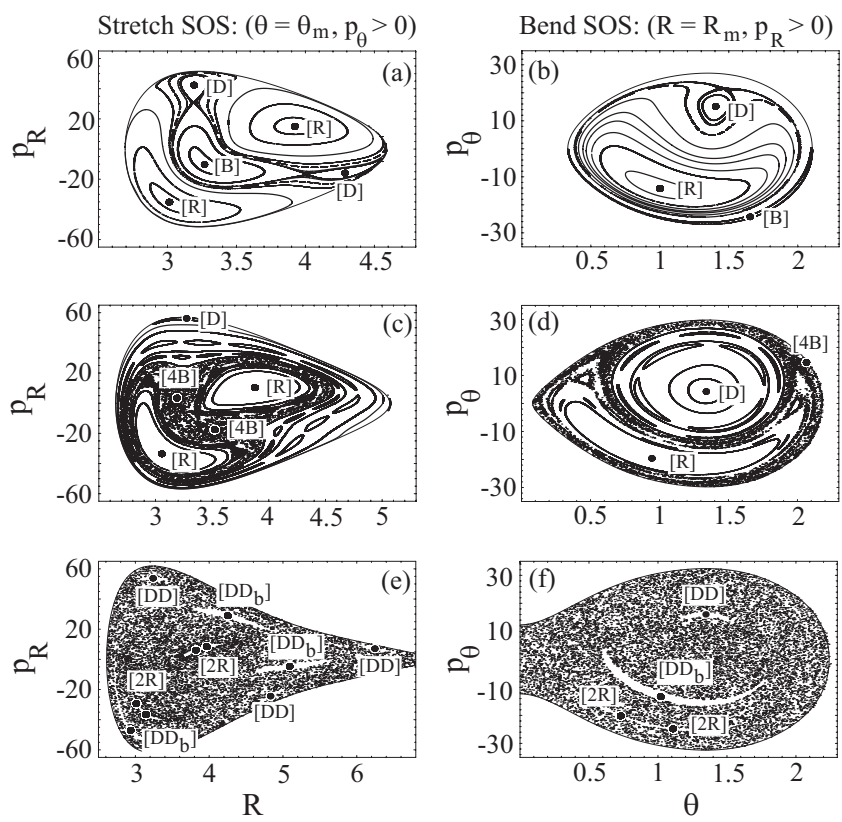

FIG. 5. Poincaré surfaces of section for (a),(b) $E=14000 \mathrm{~cm}^{-1}$, (c),(d) $E=17020 \mathrm{~cm}^{-1}$ and (e),(f) $E=20150 \mathrm{~cm}^{-1}$. Outer energy contours are calculated explicitly rather than being generated by integrating a single trajectory.

$\left(R, p_{R}\right)$ every time $\left(\theta=\theta_{m}, p_{\theta}>0\right)$. The bend SOS is obtained by plotting $\left(\theta, p_{\theta}\right)$ every time $\left(R=R_{m}, p_{R}>0\right)$. At energies below about $10000 \mathrm{~cm}^{-1}[\mathrm{R}]$ and [B] each produce a single island in each SOS. At higher energies [R] produces a two island chain in the stretch SOS as shown in Fig. 5(a). The appearance of the second island is the result of the horseshoe shape that $[\mathrm{R}]$ acquires as it reaches the 2:1 Fermi resonance [Figs 4(a) and 4(b)].

Just below $13900 \mathrm{~cm}^{-1}$ a pair of stable and unstable POs are born out of a saddle-center (sometimes referred to as a saddle-node [24]) bifurcation. The stable PO, [D], shown in Fig. 4(a), is first born as a mixture of bend and stretch motions similar to [R]. However, [D] quickly loses its bend character as its energy increases as seen in Fig. 4(b). [D] thus replaces $[\mathrm{R}]$ as the PO representing almost pure stretch motion. It is labeled " $\mathrm{D}$ " because it provides the dominant pathway leading to dissociation of $\mathrm{Cl}$ from the $\mathrm{HO}$ complex. Figures 5(a) and 5(b) show that the saddle-center bifurcation also leads to the first appearance of chaos on the scale of the SOS, as a thin layer develops around the separatrix associated with the unstable PO born at the saddle-center bifurcation. At around $14500 \mathrm{~cm}^{-1}[\mathrm{~B}]$ undergoes a period doubling bifurcation into [2B] taking on an inverted "V" shape. [2B] remains stable until just above the saddle-point energy.

\section{B. Saddle point to dissociation}

In Figs. 5(c)-5(f), we show Poincaré surfaces of section (SOS) of the stretch and bend motions for energies $17020 \mathrm{~cm}^{-1}$ and $20150 \mathrm{~cm}^{-1}$. As energy increases, [R] continues to increase its bend character until undergoing a period doubling bifurcation into [2R] [Fig. 4(c)] approximately $200 \mathrm{~cm}^{-1}$ below dissociation. [2R] remains stable up to dissociation.
Above $17010 \mathrm{~cm}^{-1}$ it becomes possible for trajectories to cross the PES saddle at $(R, \theta)=(3.16,0)$ and the bend potential becomes a double-well potential. Trajectories can begin to transition between the potential well at $\left(R_{m}, \theta_{m}\right)$ to the identical potential well at $\left(R_{m},-\theta_{m}\right)$. This causes $[2 \mathrm{~B}]$ to undergo a period doubling into [4B] executing a [2B] PO in the $\left(R_{m}, \theta_{m}\right)$ potential well followed by a $[2 \mathrm{~B}] \mathrm{PO}$ in the $\left(R_{m},-\theta_{m}\right)$ well. The $\theta>0$ portion of $[4 \mathrm{~B}]$ is shown in Fig. 4(b) with the $\theta<0$ half being a reflection about the $R$ axis. The separatrix associated with an unstable PO sitting at the PES saddle point separates trajectories that sample both potential wells and trajectories confined to a single well. The [B] family period doublings lead to a significant portion of the SOS becoming chaotic as shown in Figs. 5(c) and 5(d). As energy increases [4B] very quickly becomes engulfed in this chaotic sea. Period doublings of the [B] family of POs occur at slightly lower energies in [3]. This is attributed primarily to the lower energy saddle in their PES.

PO [D] continues to stretch radially along in the dissociation channel until going unstable through a period doubling at approximately $19800 \mathrm{~cm}^{-1}$. The period doubled orbit [2D] very quickly becomes unstable as energy increases. Just above $20000 \mathrm{~cm}^{-1}$ another saddle-center bifurcation, this time associated with a 3:1 resonance between bend and stretch motions, gives birth to a stable PO [DD]. Figure 4(c) shows that $[\mathrm{DD}]$ replaces [D] as the PO governing the dissociation channel. As energy increases [DD] continues to stretch further along the dissociation channel until going unstable approximately $100 \mathrm{~cm}^{-1}$ below dissociation. As the energy $E$ approaches the dissociation energy $D_{e}$ the frequency of stretch motions goes to zero. Bend and stretch motions thus pass through all $n: 1$ resonances as $\mathrm{HOCl}$ approaches dissociation. This suggests a possible cascade of saddle-center bifurcations. However, due to the majority of phase space becoming engulfed in chaos no further bifurcations below the dissociation energy could be located using the shooting method.

The $[\mathrm{B}],[\mathrm{R}]$, and [D] families of POs are the most physically relevant in terms of representing underlying bend and stretch motions and supporting quantum wave functions [3]. However, they are not the only POs present in $\mathrm{HOCl}$. The most significant PO not yet mentioned is $\left[\mathrm{DD}_{b}\right]$ shown in Fig. 4(c). $\left[\mathrm{DD}_{b}\right]$ is born out of a saddle-center bifurcation near $E=19650 \mathrm{~cm}^{-1}$ and remains stable up to approximately $100 \mathrm{~cm}^{-1}$ below dissociation. While $\left[\mathrm{DD}_{b}\right]$ is not present in [3], Figs. 5(e) and 5(f) show that in the present model of $\mathrm{HOCl}\left[\mathrm{DD}_{b}\right]$ has a noticeable presence in the classical phase space and could be expected to support quantum wave functions. There is also a second saddle-center bifurcation occurring at nearly the same energy and same position in phase space as the $\left[\mathrm{DD}_{b}\right]$ bifurcation however the stable PO born at this bifurcation very quickly goes unstable and is unlikely to have any influence in the quantum domain.

\section{SCATTERING DYNAMICS}

For energies above dissociation the dynamics of the $\mathrm{HO}-\mathrm{Cl}$ complex is governed by the stable and unstable manifolds associated with an unstable $\mathrm{PO}$ at $R=\infty$. As $R \rightarrow \infty$ 

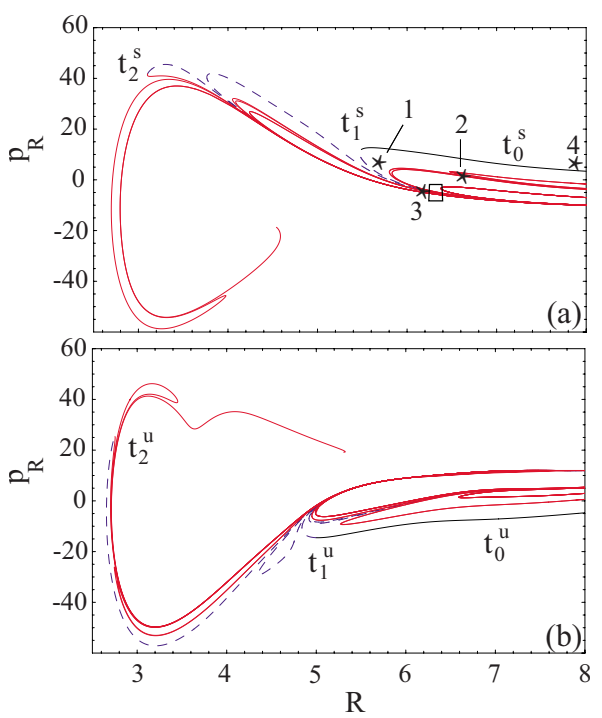

FIG. 6. (Color online) Stable (a) and unstable (b) manifolds of the PO at $\left(R=\infty, p_{R}=0\right)$ that form the homoclinic tangle at energy $E=21000 \mathrm{~cm}^{-1}$. Zeroth-, first-, and second-order tendrils, denoted $t_{0}, t_{1}$, and $t_{2}$ are shown in black, dashed blue, and red, respectively. Numbered stars in (a) indicate successive intersections of a single scattering trajectory with the SOS. The trajectory intersects the SOS three times between $t_{0}^{u}$ and $t_{0}^{s}$ indicating a step number of 3 .

(asymptotic region) $V(R, \theta) \rightarrow D_{e}$ and the Hamiltonian has a $\mathrm{PO}$ at $\left(R=\infty, p_{R}=0\right)$. The Monodromy matrix for this $\mathrm{PO}$ has all eigenvalues equal to one making the orbit locally parabolic $[14,25]$. The stable and unstable manifolds of this PO form a homoclinic tangle in phase space. To construct the tangle we add an additional intersection condition to the stretch SOS: $\left(\theta=-\theta_{m}, p_{\theta}<0\right)$. Plotting $\left(R, p_{R}\right)$ any time $\theta$ crosses $\pm \theta_{m}$ with $p_{\theta} \gtrless 0$ shows the dynamics in both potential wells in a single plot. Often it is convenient to choose the SOS to be along a symmetry line of the potential so that the stable and unstable manifolds are symmetric about $p_{R}=0$. In $\mathrm{HOCl}$ the potential hill at $\theta=0$ rises high above the dissociation energy. Thus, while the stable and unstable manifolds would be symmetric in a $\theta=0$ SOS they would also be discontinuous in the region of the potential hill. For this reason we choose the $\theta= \pm \theta_{m}$ SOS despite its lack of symmetry.

Figures 6(a) and 6(b) show the stable and unstable manifolds, respectively, which form the homoclinic tangle, in the stretch SOS at $E=21000 \mathrm{~cm}^{-1}$. This energy is chosen to be within the energy range for which quantum calculations of quasibound states have been performed $[4,16]$. The stable and unstable manifolds form a line of trajectories that separates trajectories that monotonically approach $R=\infty$ from trajectories that exhibit one or more turning points. This defining characteristic is used to locate the zeroth-order tendril of the stable $\left(t_{0}^{s}\right)$ and unstable $\left(t_{0}^{u}\right)$ manifolds. Using the Hamiltonian in Eq. (11) the zeroth-order stable (unstable) tendril is integrated backward (forward) in time to find each trajectory's next intersection with the SOS to produce the first-order tendril $t_{1}^{s}\left(t_{1}^{u}\right)$. Continuing in this way we obtain the secondorder tendrils $t_{2}^{s}$ and $t_{2}^{u}$. Already at second order the tangle displays a great deal of structure. Figure 7(a) shows an enlargement of the boxed region in Fig. 6(a). A further enlarge-
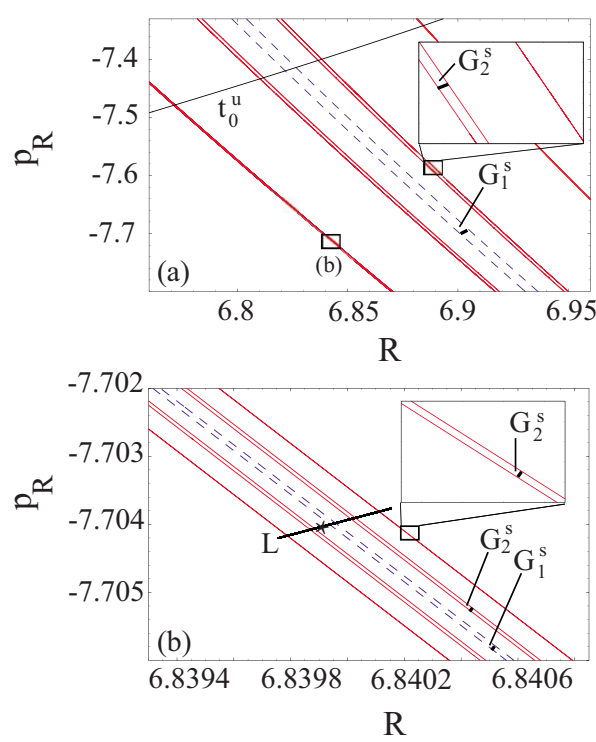

FIG. 7. (Color online) (a) Enlargement of the boxed region in Fig. 6(a). Inset shows that what appears to be a single line is actually a pair of very closely spaced lines. An enlargement of the far right-hand line in the inset would show it to be a pair of closely spaced lines as well. (b) Enlargement of the "b" box in (a) showing the central structure of (a) repeated on a smaller scale. Areas enclosed by neighboring segments of the stable manifold which are bounded above by $t_{0}^{u}$ and stretch into the asymptotic region form gaps which determine the dynamics of scattering trajectories. Firstand second-order stable gaps, $G_{1}^{S}$ and $G_{2}^{s}$, enclosed by first- and second-order stable tendrils, respectively, are indicated. Line $\mathrm{L}$ is the seventh iterate (seventh intersection with the SOS) of scattering trajectories used in Figs. 11 and 12. The star in (b) indicates the scattering trajectory shown in Fig. 6(a).

ment in Fig. 7(b) shows the central structure of Fig. 7(a) repeated in what at first appears to be a single line. The firstand second-order tendrils stretch into the asymptotic region with both tendrils exhibiting multiple folds. Figure 8 illustrates this behavior schematically showing an exaggerated sketch of the first-order stable tendril winding back and forth between the asymptotic region and smaller $R$ values. This winding is due to the potential wall at $\theta=\pi$ and the potential hill at $\theta=0$. As the stable and unstable manifolds evolve towards smaller $R$ values they are repeatedly deflected by

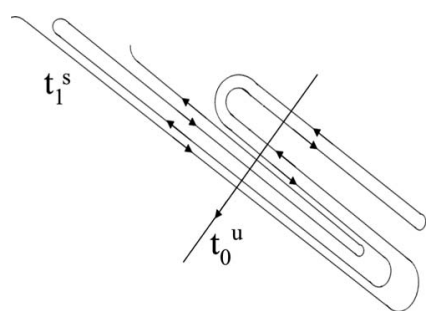

FIG. 8. Schematic of the first-order stable tendril showing multiple folds as the tendril winds between the asymptotic region and the zero-order unstable tendril. Multiple folds are observed for all energies at which the $2 \mathrm{D}$ model of $\mathrm{HOCl}$ is valid. The structure of the homoclinic tangle shows qualitative changes only for $E$ $\gtrsim 25000 \mathrm{~cm}^{-1}$. Arrows indicate the direction the tendrils evolve when integrated forward in time. 


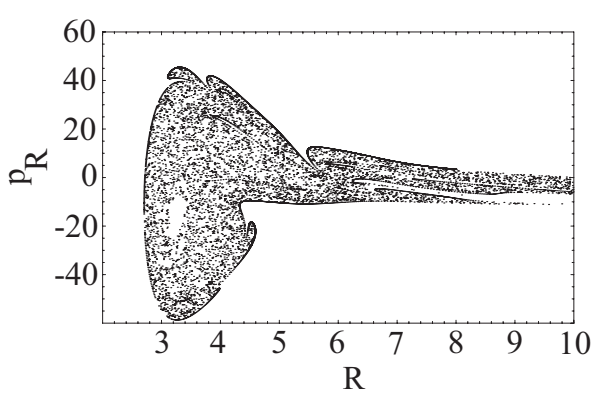

FIG. 9. SOS produced by integrating 1000 trajectories initially on the zeroth-order stable tendril backward in time through several intersections with the SOS. The large chaotic region filled by these trajectories indicates that the homoclinic tangle densely fills the majority of the energetically accessible phase space except for a small region of stability surrounding $\mathrm{PO}\left[\mathrm{B}_{0}\right]$ at $\left(R=3.26, p_{R}\right.$ $=-14.55)$.

collisions with these potential barriers. These deflections dominate the structure of the homoclinic tangle. Similar behavior has been observed in coupled Morse and harmonic oscillators [26] and for an electron scattering off a magnetic dipole [12].

Beyond second order, the tendrils become too complicated and too closely spaced to be adequately resolved in a SOS. In Fig. 9 we follow 1000 trajectories, initially lying on the zeroth-order stable tendril, that are integrated backward in time through approximately 20 intersections with the SOS. Although none of the detailed structure of the stable manifold can be resolved, Fig. 9 shows that the homoclinic tangle densely fills the majority of the energetically accessible phase space except for a small island of stability near $\left(R, p_{R}\right)=(3.26,-14.55)$ and an infinity of smaller surrounding islands not visible on the scale of the SOS. The stable PO at the center of this island, $\left[\mathrm{B}_{0}\right]$, is shown in Fig. 10(a) and represents chlorine oscillating back and forth across the PES saddle between the two potential wells at positive and negative values of $\theta$. [ $\left.\mathrm{B}_{0}\right]$ is born out of a saddle-center bifurcation just below the dissociation energy and exists for all energies up to above $25000 \mathrm{~cm}^{-1}$. The area of the stable region
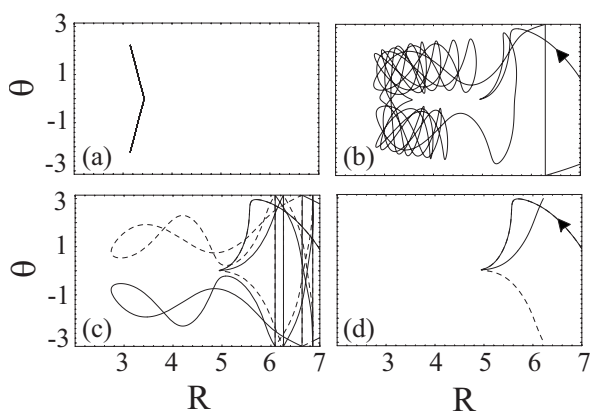

FIG. 10. (a) PO $\left[\mathrm{B}_{0}\right]$ at the center of the region of stability in Fig. 9. (b) A typical scattering trajectory passing several times over the PES saddle connecting potential wells at positive and negative values of $\theta$. (c) Symmetric trajectories (one solid one dashed) indicated by arrows in Fig. 12. (d) Same as (c) showing trajectories only just before and just after collision with $\theta=0$ potential barrier. The solid and dashed trajectories are deflected symmetrically upward and downward, respectively, from the potential barrier. surrounding $\left[\mathrm{B}_{0}\right]$ in the stretch SOS ranges from 1 to 2 times Planck's constant for $22000 \mathrm{~cm}^{-1} \lesssim E \lesssim 23300 \mathrm{~cm}^{-1}$. In the bend SOS the stable region is a thin crescent shape with an area of approximately Planck's constant in this same energy range. Given the volume of this stable region in phase space $\left[\mathrm{B}_{0}\right]$ is expected to support long-lived quasibound states.

Probing the structure of the homoclinic tangle beyond second order requires the use of scattering functions. We examine two important examples of scattering functions: Time delay and asymptotic HO angular momentum. Time delay, $\delta T$, is the amount of time the incoming chlorine atom spends "caught" in the homoclinic tangle before returning to the asymptotic region. We define $\delta T$ as

$$
\delta T=T-\frac{\mu_{1}\left(R_{\text {in }}-8\right)}{\left|p_{R, \text { in }}\right|}-\frac{\mu_{1}\left(R_{\text {out }}-8\right)}{\left|p_{R, \text { out }}\right|},
$$

where $T$ is the time for the chlorine atom to approach from the asymptotic region, scatter and return to the asymptotic region. Because we are interested only in the time spent scattering, that is the time spent caught in the tangle, we subtract the times spent traversing the asymptotic region. Defining the asymptotic region as $R>8$ these times are given by $\frac{\mu_{1}\left(R_{\text {in }}-8\right)}{\left|p_{R, \text { in }}\right|}$ and $\frac{\mu_{1}\left(R_{\text {out }}-8\right)}{\left|p_{R, \text { out }}\right|}$ where $p_{R \text {,in }}$ and $p_{R \text {,out }}$ are the values of $p_{R}$ when $R$ crosses $R_{\text {in }}$ and $R_{\text {out }}$ (defined below).

The asymptotic HO angular momentum is the angular momentum of the $\mathrm{HO}$ complex after the chlorine atom has scattered and returned to the asymptotic region. The angular momentum of $\mathrm{HO}$ relative to its center of mass is given simply by $\mathbf{L}_{2}=p_{\theta} \hat{\mathbf{y}}$. To see how time delay and the HO angular momentum vary from one scattering trajectory to another we take a collection of initial conditions in the asymptotic region each with $R_{\text {in }}=R_{\text {out }}=12, p_{R \text {, in }}=-2, p_{\theta}$ $=5.9726$ and a range of value of $\theta$ that is specified below. This corresponds to scattering trajectories starting just outside (below) the unstable manifold, each with $E$ $=21000 \mathrm{~cm}^{-1}$. Each initial condition can then be uniquely labeled by its reduced phase [25],

$$
\chi=\theta-\frac{R p_{\theta} \mu_{1}}{p_{R} r_{o}^{2} \mu_{2}}=\theta-\frac{R}{p_{R}} \mu_{1} \omega,
$$

where $\omega \equiv \frac{\partial H}{\partial p_{\theta}}$ as $R \rightarrow \infty$. The reduced phase is the phase difference between motion in the $\theta$ and $R$ directions. Because energy, $p_{R}, p_{\theta}$, and $\chi$ are all conserved quantities under $H$ in the asymptotic region, the structure of time delay and HO angular momentum is independent of the choice of $R_{\text {in }}$ and $R_{\text {out }}$ as long as they both lie in the asymptotic region.

Because $\chi$ and $\chi+2 \pi$ describe the same initial condition, time delay and HO angular momentum are functions of $\chi \bmod 2 \pi$. We focus on a narrow range of $\chi$ corresponding to $-1.73165 \leqslant \theta \leqslant-1.73036$ for which the scattering trajectories, when integrated forward in time, cut completely across a bundle of stable tendrils as shown in Fig. 7(b). Figure 11 shows the asymptotic HO angular momentum and 

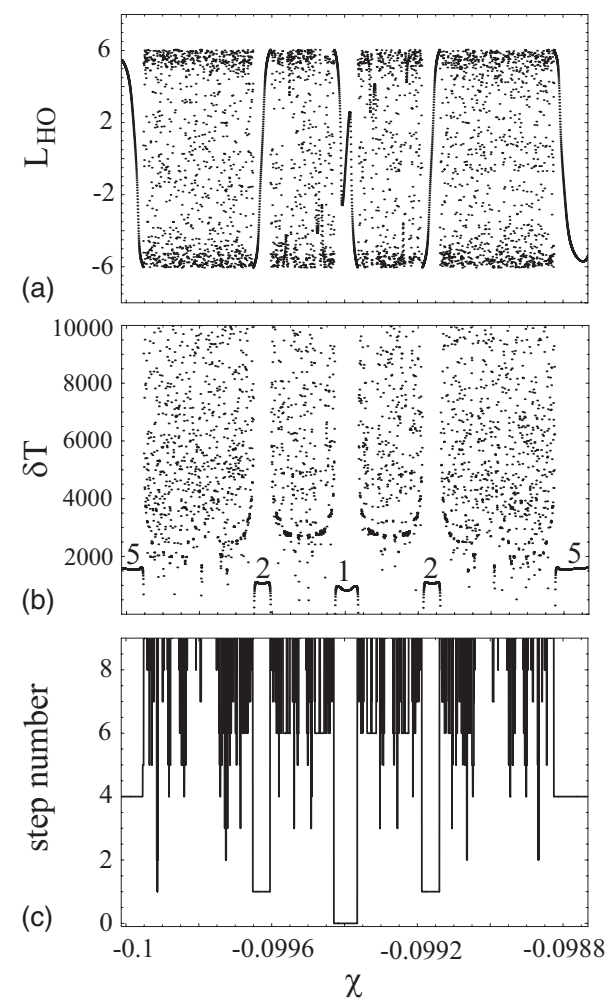

FIG. 11. (a) Asymptotic HO angular momentum, (b) time delay, and (c) step number for 5000 scattering trajectories comprising line L in Fig. 7(b). Regions of continuity in $L_{\mathrm{HO}}$ and $\delta T$ occur at the same intervals of $\chi$ indicating that every scattering function contains the same fractal structure corresponding to the set of intersections between the stable manifold and the zero-order unstable tendril. Given the step number for any trajectory the order of the gap the trajectory sits in Fig. 7(b) is given simply by (step number +1 ). In this way all regions of continuity in $\delta T$ are labeled by what order stable gap the trajectories initially sit in.

time delay for these trajectories. For certain ranges of reduced phase, both $L_{\mathrm{HO}}$ and $\delta T$ vary continuously while for other ranges both vary erratically. Both $L_{\mathrm{HO}}$ and $\delta T$ contain singularities at values of $\chi$ for which the incoming trajectory intersects the stable manifold. These "singular" trajectories become caught in the homoclinic tangle forever having an infinite delay time and an undefined asymptotic HO angular momentum because the trajectory never returns to the asymptotic region. The singularities in plots of $L_{\mathrm{HO}}$ and $\delta T$ have the same structure as the set of intersections between $t_{0}^{u}$ and the stable manifold. The singularities form a fractal set with measure zero.

The structure of the homoclinic tangle can be characterized by the gaps formed by intersections of tendrils of the stable manifold with the zeroth-order unstable tendril. An $n$ th-order stable gap, sometimes referred to as a lobe or turnstile, is the area enclosed by the $n$ th-order stable tendril and the zeroth-order unstable tendril. First- and second-order stable gaps are illustrated in Fig. 7. Because the $n$ th-order stable tendril is formed by integrating each trajectory on the $(n-1)$ th-order stable tendril backward in time and finding each trajectory's next intersection with the SOS it is not surprising that every scattering trajectory that intersects the SOS inside an $n$ th-order stable gap, when integrated forward in time, will have its next intersection with the SOS inside an $(n-1)$ th-order stable gap [27]. If a scattering trajectory initially lies within an $n$ th-order stable gap it moves to an $(n$ -1)th-order stable gap on each successive intersection with the SOS. Eventually intersecting the SOS inside the firstorder stable gap, the trajectory's next intersection with the SOS will be above the zeroth-order stable tendril signaling the trajectory's return towards the asymptotic region. The scattering dynamics of $\mathrm{Cl}$ is completely determined by the gap structure formed by the stable manifold. This gap structure is most easily investigated by constructing a step number for a collection of scattering trajectories. The step number counts the number of times a trajectory intersects the SOS in between the trajectory's last intersection below $t_{0}^{u}$ and its first intersection above $t_{0}^{s}$. This is illustrated in Fig. 6(a) for a scattering trajectory with step number $=3$. When we integrate this trajectory forward in time it intersects the SOS three times above $t_{0}^{u}$ before intersecting above $t_{0}^{s}$.

Figure 11(c) shows step number as a function of reduced phase for the trajectories comprising line L in Fig. 7(b). Because a trajectory moves from a $n$ th-order stable gap to a $(n-1)$ th-order stable gap on each "step" a trajectory with step number $=n$ must lie in a stable gap of order $n+1$ in Fig. 7(b). Higher-order steps in Fig. 11(c) thus reveal gaps formed by higher-order tendrils which cannot be resolved in a SOS. Comparing time delay and step number shows that every continuous region in time delay corresponds to a given step in step number. Using step number, any continuous region in time delay (or any other scattering function) can be labeled by what order gap the trajectories originate. Examining ever smaller ranges of $\chi$ with ever higher resolution will reveal smaller regions of continuity corresponding to smaller gaps, ad infinitum.

We return to the importance of the potential barriers at $\theta=\pi$ and $\theta=0$ by considering which potential well, $\theta=+\theta_{m}$ or $\theta=-\theta_{m}$, scattering trajectories first enter. Because $\mathbf{L}_{\text {tot }}$ $\equiv 0$ the $\mathrm{HO}$ complex will rotate if the chlorine approaches with a nonzero impact parameter (nonzero angular momentum). If the incoming chlorine meets the HO complex end on, $\theta=\pi$ or $\theta=0$, the chlorine may be completely reflected or may scatter off one or both potential barriers before entering a potential well. For each scattering trajectory we plot $\theta$ when the trajectory first crosses $R=3.75$. The resulting Fig. 12 shows a fractal structure which is symmetric about the center with $\theta \rightarrow-\theta$. This symmetry can be easily understood by looking at a pair of symmetric trajectories. Figures 10(c) and 10(d) show one such pair of trajectories indicated by arrows in Fig. 12. Initially the trajectories are almost identical however upon collision with the $\theta=0$ potential barrier one trajectory is deflected upwards and the other downwards. Because the PES is symmetric about $\theta=0$ the trajectories continue to evolve identically differing only in the sign of $\theta$. The scattering trajectory exactly in the center of Fig. 12 [the midpoint of line L in Fig. 7(b)] is reflected from the $\theta=0$ barier parallel to the $\theta$ axis. All other scattering trajectories occur in symmetric pairs.

By considering total angular momentum zero and the $\mathrm{HO}$ bond length held fixed $\mathrm{HOCl}$ is reduced to two degrees of freedom. In systems with more degrees of freedom the cha- 


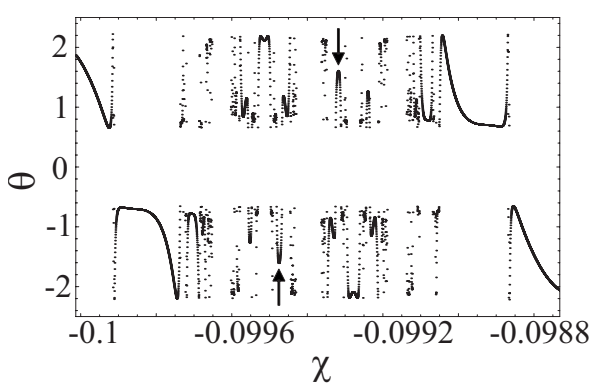

FIG. 12. The value of $\theta$ when each scattering trajectory first crosses $R=3.75$. The blank region near $\chi=-0.0994$ and smaller regions near $\chi=-0.09965$ and $\chi=-0.09915$ correspond to trajectories in first- and second-order gaps, respectively, all of which are reflected by the potential barrier at $\theta=0$ never entering either potential well. Arrows indicate a pair of symmetric trajectories shown in Figs. 10(c) and 10(d).

otic scattering in phase space is directed by the stable and unstable manifolds associated with normally hyperbolic invariant manifolds [28]. These form dividing surfaces between trajectories of qualitatively different scattering behavior [29]. These structures are the higher dimensional counterparts of the fixed points and their stable and unstable manifolds in our 2D Poincaré surfaces of section.

\section{CONCLUSIONS}

Below dissociation the $\mathrm{HO}-\mathrm{Cl}$ dissociation channel is governed by periodic orbits born out of saddle-center bifurcations. These bifurcations giving rise to the $[\mathrm{D}]$ and [DD] periodic orbits have been associated with the formation of new quantum states with probabilities localized along the dissociation channel [3].

Above dissociation the homoclinic tangle that determines the $\mathrm{HO}+\mathrm{Cl}$ scattering dynamics has a very convoluted and somewhat constricted structure due to the high potential walls in the $\mathrm{HO}-\mathrm{Cl}$ potential energy surface forming a narrow entrance channel for the incoming chlorine atom. The high potential walls cause reflections of the $\mathrm{Cl}$ off the $\mathrm{HO}$ complex and multiple folds in the homoclinic tangle. The narrow entrance channel causes the internal structure of the homoclinic tangle to be compressed and difficult to resolve numerically.

The phase space above dissociation is dominated by the homoclinic tangle and chaos that stretch well into the asymptotic region. However, embedded in the chaotic sea, there exists a stable bendlike periodic orbit and a surrounding island structure that classically cannot dissociate, even above the dissociation energy. Quantum mechanically this structure appears to be large enough to support quantum states that will have fairly long lifetimes, but will eventually tunnel out and cause dissociation. The structure and lifetime of quantum mechanical quasibound states in $\mathrm{HO}-\mathrm{Cl}$ and their connection to the classical dynamics presented here will be investigated in a future paper.

\section{ACKNOWLEDGMENTS}

The authors wish to thank R. Schinke for providing access to the PES used in this study. A.M.B. and L.E.R. thank the Robert A. Welch Foundation (Grant No. F-1051) for partial support of this work. C.J. thanks CONACyT (Grant No. 43375) and DGAPA (Grant No. IN-107308) for their support.
[1] L. E. Reichl, The Transition to Chaos, 2nd ed. (SpringerVerlag, New York, 2004).

[2] M. Joyeux, S. Y. Grebenshchikov, J. Bredenbeck, R. Schinke, and S. Farantos, Adv. Chem. Phys. 130, 267 (2005).

[3] M. Joyeux, D. Sugny, M. Lombardi, R. Jost, R. Schinke, S. Skokov, and J. Bowman, J. Chem. Phys. 113, 9610 (2000).

[4] J. Weiß, J. Hauschildt, S. Yu Grebenshchikov, R. Düren, R. Schinke, J. Koput, S. Stamatiadis, and S. C. Farantos, J. Chem. Phys. 112, 77 (2000).

[5] R. Jost, M. Joyeux, S. Skokov, and J. Bowman, J. Chem. Phys. 111, 6807 (1999).

[6] A. Emmanouilidou and L. E. Reichl, Phys. Rev. A 65, 033405 (2002).

[7] K. Na, D. Jarukanont and L. E. Reichl, Phys. Rev. E 77, 046208 (2008).

[8] N. Ben-Tal, N. Moiseyev, and R. Kosloff, J. Chem. Phys. 98, 9610 (1993).

[9] D. Jarukanont, K. Na, and L. E. Reichl, Phys. Rev. A 75, 023403 (2007).

[10] C. Jung and H. J. Scholz, J. Phys. A 20, 3607 (1987).

[11] T. Tel, Phys. Rev. A 36, 1502 (1987).

[12] B. Rückerl and C. Jung, J. Phys. A 27, 6741 (1994).

[13] E. Okon, W. Parker, W. Chism, and L. E. Reichl, Phys. Rev. A
66, 053406 (2002).

[14] A. Emmanouilidou, C. Jung, and L. E. Reichl, Phys. Rev. E 68, 046207 (2003).

[15] T. Timberlake and L. E. Reichl, Phys. Rev. A 64, 033404 (2001).

[16] J. Hauschildt, J. Weiß, C. Beck, S. Yu Grebenshchikov, R. Düren, R. Schinke, and J. Koput, Chem. Phys. Lett. 300, 569 (1999).

[17] H. Lee, C. Jung, and L. E. Reichl, Phys. Rev. B 73, 195315 (2006).

[18] H. Goldstein, Classical Mechanics (Addison-Wesley, Reading, 1980).

[19] S. Skokov, K. A. Peterson, and J. M. Bowman, Chem. Phys. Lett. 312, 494 (1999).

[20] K. Peterson, S. Skokov, and J. Bowman, J. Chem. Phys. 111, 7446 (1999).

[21] S. C. Farantos, Int. Rev. Phys. Chem. 15, 345 (1996).

[22] E. Reithmeier, Periodic Solutions of Nonlinear Dynamical Systems (Springer, Berlin, 1991).

[23] M. A. M. Aguiar, C. P. Malta, M. Baranger, and K. T. R. Davies, Ann. Phys. 180, 167 (1987).

[24] J. M. Mao and J. B. Delos, Phys. Rev. A 45, 1746 (1992).

[25] C. Jung, G. Orellana-Rivadeneyra, and G. A. Luna-Acosta, J. 
Phys. A 38, 567 (2005).

[26] A. Zembekov, J. Chem. Phys. 101, 8842 (1994).

[27] S. Wiggins, Chaotic Transport in Dynamical Systems (Springer-Verlag, New York, 1992).
[28] S. Wiggins, Normally Hyperbolic Invariant Manifolds in Dynamical Systems (Springer-Verlag, New York, 1994).

[29] T. Uzer, C. Jaffe, J. Palacian, P. Yanguas, and S. Wiggins, Nonlinearity 15, 957 (2002). 